\title{
Augmented reality visualization for aiding biopsy procedure according to computed tomography based virtual plan
}

\author{
MARCIN MAJAK ${ }^{1}$, MAGDALENA ŻUK ${ }^{1}$, EWELINA ŚWIĄTEK-NAJWER ${ }^{1}$, \\ MICHAŁ POPEK ${ }^{1}$, PIOTR PIETRUSKI ${ }^{2}$ \\ ${ }^{1}$ Wrocław University of Science and Technology, Faculty of Mechanical Engineering, \\ Wrocław, Poland. \\ ${ }^{2}$ Department of Plastic Surgery, Prof. W. Orlowski Memorial Hospital, \\ Medical Centre of Postgraduate Education, Warsaw, Poland.
}

\begin{abstract}
Purpose: The purpose of this study was to develop and verify an intraoperative module for supporting navigated biopsy procedures using optical see-through head-mounted display (HMD). Methods: Biopsy procedure including entry and endpoints of needle insertion was planned preoperatively having regard to the resection region segmentation and safety margin definition. Biopsy procedures were performed by two users using an intraoperative optical navigation module on a specially prepared brain phantom. Two visualization techniques were compared: an accurate augmented reality one, where a virtual plan is superimposed onto surgical field by using optical see-through HMD together with personalized calibration method and visualization on the external display. Results: Averaged errors from 24 trials using external display were $2.04 \pm 0.83 \mathrm{~mm}$ for the first user and $2.69 \pm 1.11 \mathrm{~mm}$ for the second one, while applying HMD 2.50 $\pm 0.93 \mathrm{~mm}$ (the first user) and $2.17 \pm 0.82 \mathrm{~mm}$ (the second user), respectively. Conclusions: Proper usage of HMD visualization preceded by the personalized calibration allows the user to perform navigated biopsy procedure with comparable accuracy to its equivalent with the external display. Additionally, augmented reality visualization improves ergonomics and enables focusing on the surgical field without losing a direct line of sight with the field of view as it happens for external displays. However, ensuring high accuracy of augmented reality visualization still requires proper calibration and some user experience, which is challenging.
\end{abstract}

Key words: biopsy, augmented reality, computer aided surgery, head mounted display, computer navigation

\section{Introduction}

In recent years, we have observed huge development in the area of head-mounted displays (HMD) and their potential usage in many domains such as entertainment, industry, and simulated training [18]. One can divide HMD applications into two main purposes considering how content is presented: virtual reality (VR) and augmented reality (AR). The former creates an artificial (computer-generated) environment to inhabit, while the latter processing unit renders 3D objects superimposed over a user's view of the real world. Previous generations of HMDs suffered from huge latency and very small field of view, but cur- rently these limitations are significantly improved. Each new commercially available device excels display resolution and ergonomics. As a result, HMD can be successfully applied in the very demanding healthcare industry, especially in surgery applications. Since 2017, many researchers have devoted their work to adjust HMD to the clinical practice, constantly optimizing medical content presentation to benefit from direct and intuitive real-time visualization of anatomy, surgical plans, or specific surgery hints [1], [2]. According to [24], HMDs were successfully applied with image guidance and augmented reality, mostly in urology and neurosurgery specialties. Other papers report HMDs' various usage ranging from orthopedic surgeries [19], [25], in biopsy procedures for endovascular aortic,

* Corresponding author: Magdalena Żuk, Wrocław University of Science and Technology, Faculty of Mechanical Engineering, ul. Łukasiewicza 7/9, 50-371, Wrocław, Poland, e-mail:magdalena.zuk@pwr.edu.pl

Received: January 23rd, 2021

Accepted for publication: March 29th, 2021 
precise screw placement [2], [10], [27], [28] or even in very challenging cranio-maxillofacial surgeries [3], [4]. HMDs are integrated into Computer Aided Surgery (CAS) systems as two main types: optical seethrough (OST) or a video see-through (VST) [24], [29]. The first type gains much more attention recently since the user can also depend on its visual world's perception, which freely adds an additional verification step. In the simplest case, OST HMD can be used to display virtual planning (VP) content instead of previously used external displays (ED). Despite some evident constraints, since the presented VP is an only a $2 \mathrm{D}$ view, there are some advantages such as displayed data can be adjusted to the specific surgical procedure, and the operator does not lose direct contact with the operating field (OF) [23]. The second application of OST HMD is related to presenting 3D content from the first-person perspective, where VP content is perceived as spatially aligned with real objects seen in the field of view [22]. This solution is tempting, but it also has some requirements and even limitations. Firstly, we need to navigate surgical field and HMD device using an external tracking system such as optical, or magnetic navigation, inertial systems or solutions based on image processing [6], [7]. Secondly, a personalized calibration is required to ensure that $3 \mathrm{D}$ objects are perceived at the proper depth. This procedure is quite challenging, it must be ergonomic and user-friendly while ensuring the highest possible accuracy [1]. There are different algorithms for HMD calibration ranging from manual, semi-automatic, and automatic approaches [12]. Successful calibration measures the user's eyes position in reference to the tracking system's coordinate frame. As a golden standard for HMD calibration SPAAM algorithm is taken with many further improvements and modifications [26]. It assumes collecting at least six 2D-3D point correspondences used to calculate projection matrices. Manual calibration can be tedious and prone to error, so each new proposed algorithm strives to minimize the number of correspondences to collect. The semi-automatic approach is usually divided into off-line (a viewpoint camera for off-line parameter estimation) and online (where the user has to align few calibration markers) steps [17]. Finally, fully automatic calibration procedures are an alternative in which manual data collection is replaced by some external processing unit (for example, eyetracking module) [12], [13]. Automatic calibration gives very promising results and speeds up the whole procedure. However, it has one major drawback because the HMD device is more complex, which highly decreases ergonomics and usability [12].
The purpose of this work was to develop an intraoperative module for supporting navigated biopsy procedures using AR head mounted display. This functionality is a part of the MentorEye system designed for planning and aiding complex oncological treatment [21], [22]. In this work, we tried to apply OST HMD according to the second scenario for presenting 3D data from VP to support guided biopsy. To accomplish this task, we have proposed a two-stage procedure where in the adjustment phase whole 3D content from VP is superimposed on the operating field to help the user with an initial biopsy location. Later, for precise biopsy alignment, VP 3D content is minimized, and the user follows a specific surgery guide along with the color-coded and textual information. In order to verify HMD ergonomics and its usability for biopsy support in the MentorEye system, we have prepared a validation procedure with a specially designed phantom. It should be emphasized that the proposed biopsy procedure is universal and does not depend on any HMD device model or tracking system.

The structure of this paper is as follows: Section 2 presents the whole system and proposed biopsy procedure in detail. Verification results are placed in paragraph 3 along with discussion in Section 4. The whole study is concluded in Section 5 with propositions for future improvements.

\section{Materials and methods}

\section{MentorEye system}

MentorEye is a complex system providing both: virtual planning for different surgery scenarios with safety margins and VP realization under the control of the optical navigation system. The first step is based on imaging data (CT) collected before surgery. Later, three imaging projections are created (axial, coronal, sagittal) along with 3D models. Biopsy planning is preceded by resection region segmentation and safety margin definition. Using this information, entry and endpoints of a single biopsy are planned and exported into VP. The intraoperative module starts with tools selection and image data to patient registration. Finally, VP surgery guidance can be presented using ED or HMD.

\section{Personalized HMD calibration}

An accurate AR biopsy visualization in MentorEye is preceded by a personalized HMD calibration proce- 


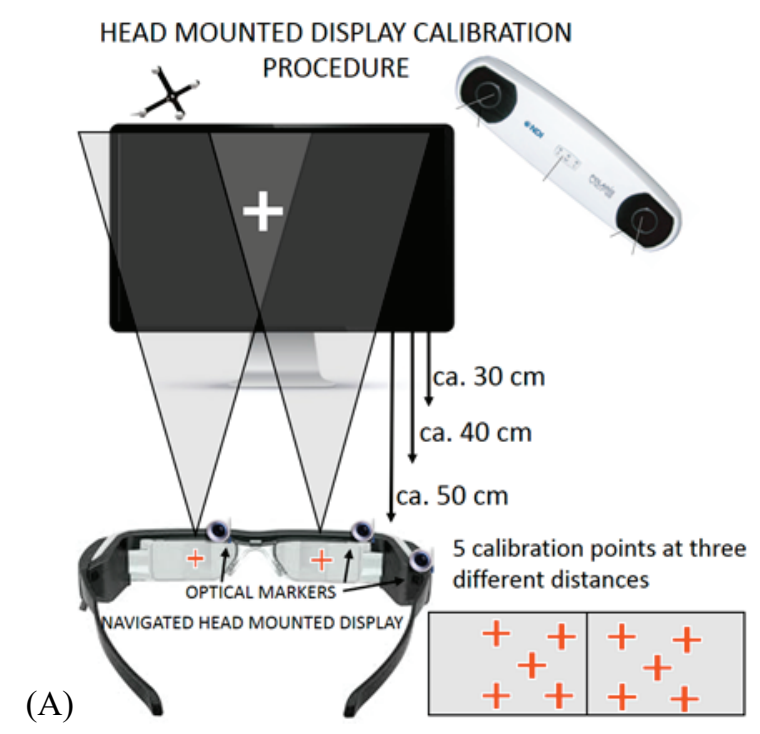

\section{AR GUIDED BIOPSY USING STEREOSCOPIC VISUALIZATION- GENERAL CONCEPT}

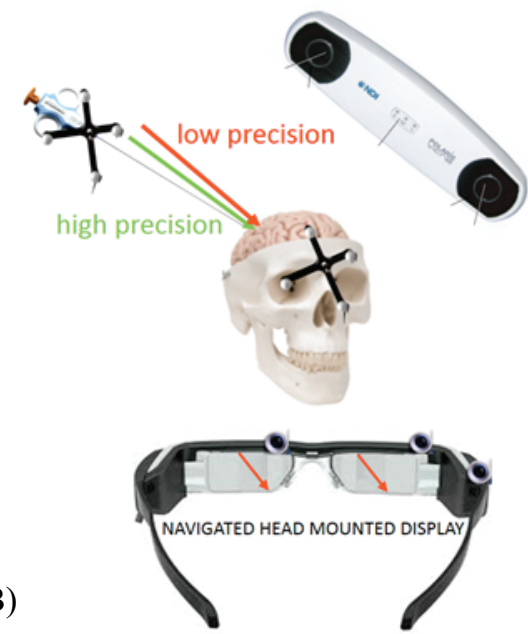

Fig. 1. General concept of HMD calibration (A) and AR intraoperative visualization (B)

dure (Fig. 1A). The user is instructed to overlay 2D objects displayed on HMD with objects presented on the calibration screen (CS). In this work, calibration was performed for left and right HMDs' display separately using three different distances from CS. Two projection matrices were determined, obtaining 15 2D-3D point pairs per eye with the SPAAM algorithm. We have implemented this approach due to its versatility and weak dependency on the hardware: requiring only attached reference frames to both HMD and CS and a tracking system for navigation. SPAAM algorithm is universal and can easily be integrated into most OST HMD devices with open SDK. What is more, it does not require specific domain knowledge from the user, so only a short introduction is sufficient.

\section{AR visualization for aiding biopsy procedure in MentorEye system}

The general concept of an AR-guided biopsy using OST HMD is shown in Fig. 1B. The navigation of the biopsy needle, as well as HMD, enables stereoscopic $3 \mathrm{D}$ visualization of CT-based virtual plan inside the surgical operating field.

Our initial tests revealed that in many cases, direct imposition of the VP components onto OF was erroneous, causing incorrect biopsy long axis introduction. Because of these reasons, we have decided to undertake an alternative path where HMD is used as a supporting tool displaying 3D surgery guide (rectangle with two circles) with color-coded and textual information about the digital coordinate system of biopsy needle against registered VP (Figs. 2, 3B). In this approach depth accuracy of perceived $3 \mathrm{D}$ models is not so crucial.

The whole biopsy procedure is divided primarily into two steps. In the first one, the user can follow 3D models representing biopsy tool along with whole VP data to initially adjust both biopsy trajectory and an entry point (Fig. 4). In the second stage, when an introductory point and long axis angle are essential to determine precisely, the user can switch off 3D models and rely only
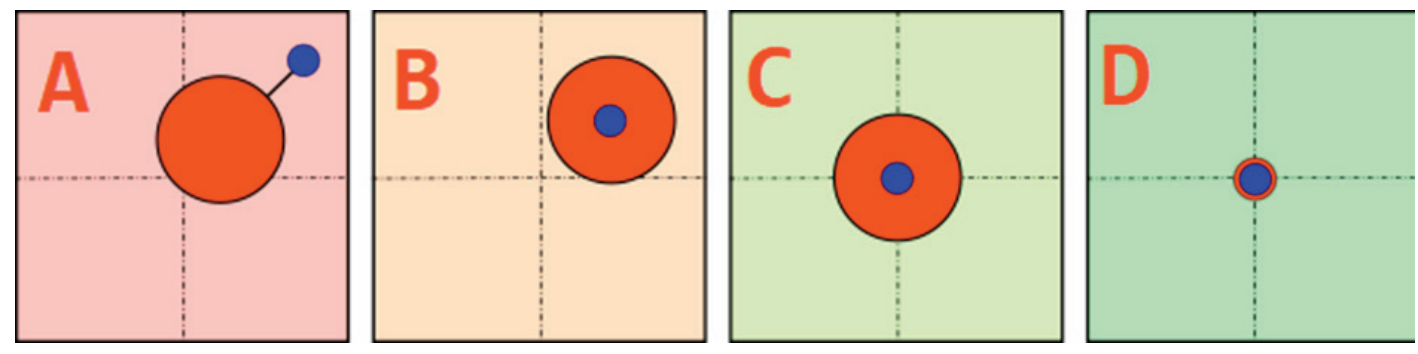

Fig. 2. MentorEye biopsy guide idea: A) incorrect position and angle, B) incorrect position, correct angle

C) incorrect depth, correct angle, D) correct depth and angle.

Background color indicates current biopsy state comparing to the virtual plan:

red-incorrect, green-acceptable accuracy threshold 
on 3D surgery guide and textual data. To indicate to the user that an acceptable entry point and long axis angle were obtained MentorEye system changes colors from red into green in both: planned 3D biopsy model and a special biopsy surgery guide.

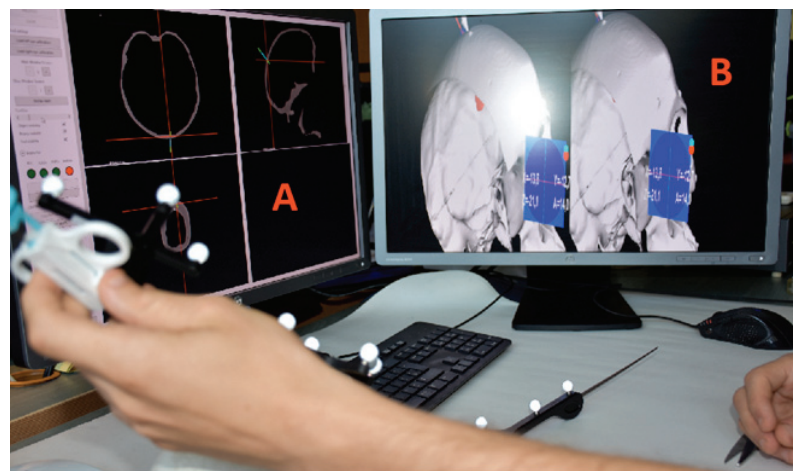

Fig. 3. MentorEye system during intra-operative biopsy navigation: A) three DICOM projections, B) 3D views generated for left and right eye displays in HMD, respectively

\section{Biopsy procedures performed on skull and brain phantom}

To validate the proposed AR solution, we have designed a special phantom comprising of skull and brain (Fig. 5). The brain model was prepared from synthetic material and divided into two parts. In the middle one, four holes (with a diameter of $10 \mathrm{~mm}$ ) were created and filled with the substance that remains its shape when a biopsy needle is successfully introduced according to the planned trajectory.

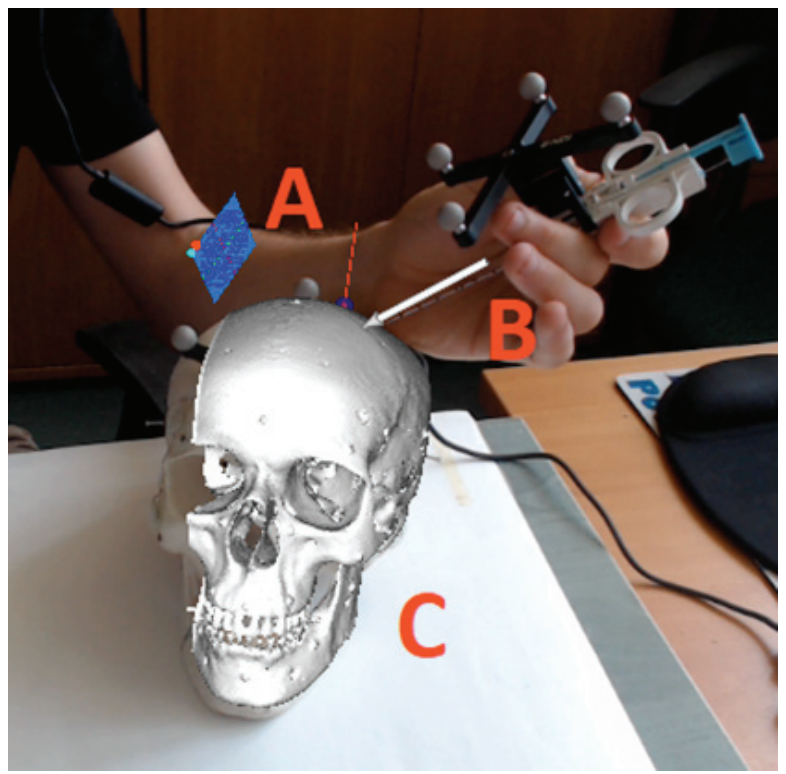

Fig. 4. Full AR view (first procedure step) seen from user's perspective, A) 3D guide + model of planned biopsy, B) 3D model of biopsy tool, C) superimposed 3D phantom model

The CT scans of the phantom were acquired (Somatom Sensation 16; Siemens Medical Solutions, Erlangen, Germany), and the image data $(512 \times 512$-pixel dataset, $0.625-\mathrm{mm}$ slice thickness) was recorded as Digital Imaging and Communications in Medicine (DICOM) files and exported to the MentorEye sys-
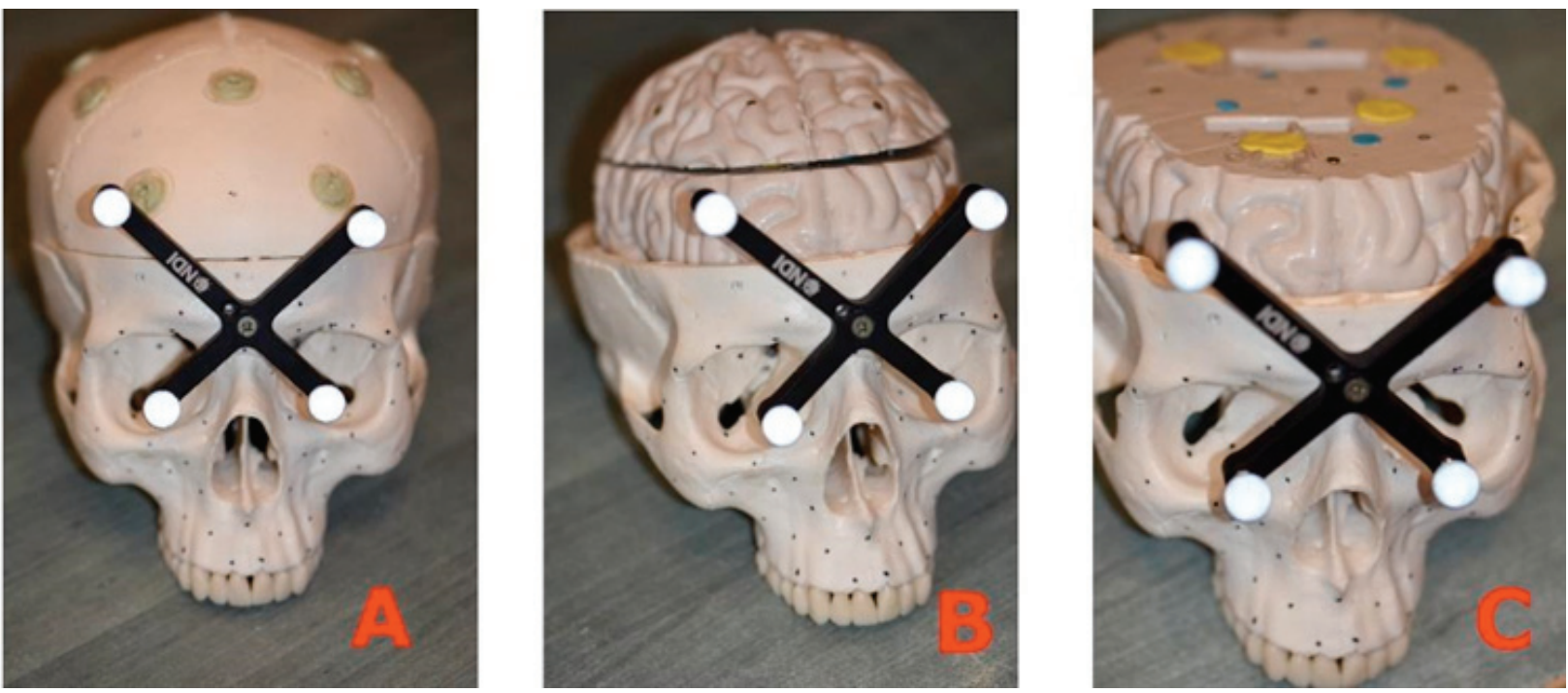

Fig. 5. Validation phantom with skull and brain (A - closed skull phantom, B - brain inside skull phantom, $\mathrm{C}$-visible structures for brain biopsy) 

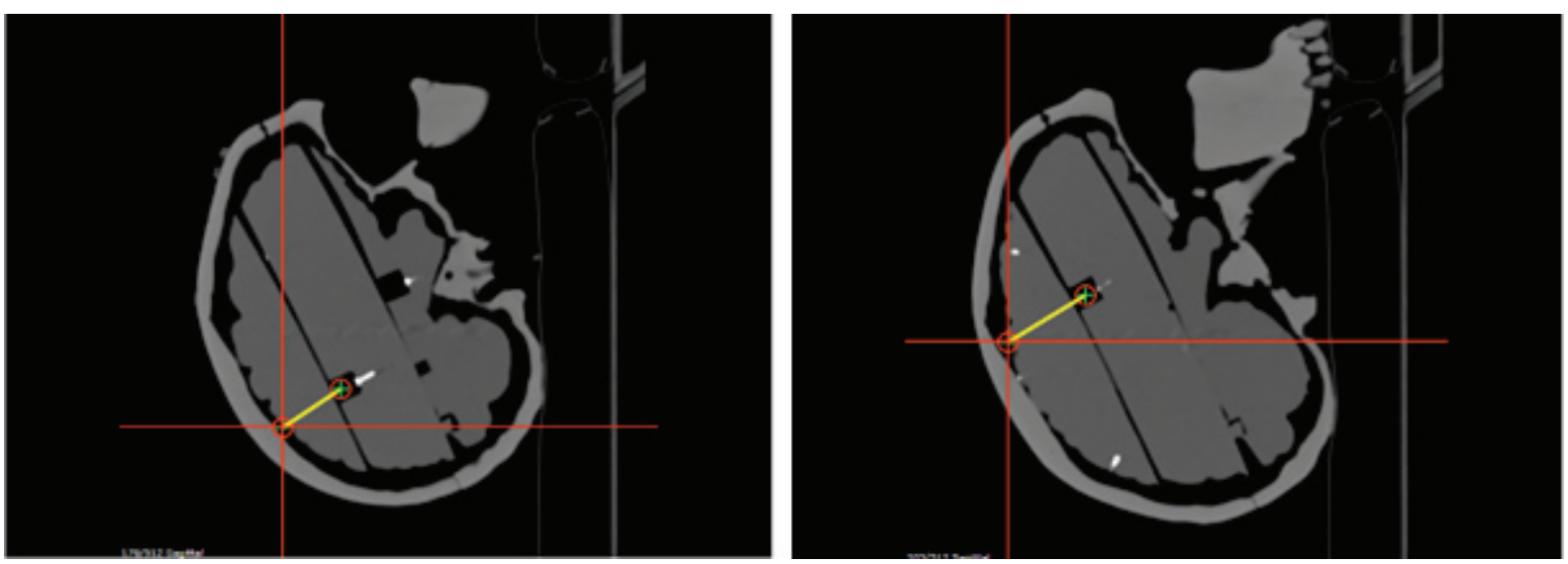

Fig. 6. Two examples of planned virtual biopsies

tem. Then 12 different biopsies were planned with a various entry point and long axis settings (in Fig. 6, two examples of planned biopsy are presented).

Two users (familiarized with AR technology) participated in the validation phase in which an infrared optical tracking system (Polaris Spectra by NDI, Canada) was used for navigation and as HMD display Epson Moverio BT-200 Smart Glasses was applied. The measurement protocol (MP) was as follows:

1. upload VP into an intraoperative navigation module of the MentorEye system,

2. pivot pointer,

3. choose OST HMD, or ED navigated biopsy mode,

4. perform personalized SPAAM calibration (Fig. 1A) if AR mode is active,

5. calibrate biopsy tool,

6. register VP with real data using seven landmark points located on the phantom's skull surface,

7. check target registration error using eight titan markers (clearly visible also in CT data) from the brain's surface,

8. perform navigated biopsy in AR or ED mode (Fig. 1B).

One should note that biopsy procedures can be done using different tools ranging especially in length. Moreover, a tracking adapter can be attached to the selected tool in various ways changing the local coordinate system. Due to these reasons, we have implemented a universal biopsy tool calibration algorithm which defines tool's tip offset and long axis in the reference to the attached tracking adapter. In Figure 7, proposed calibration steps are presented. In this case, the calibrated pointer was used to indicate four points and later collected data were used to generate a transformation matrix describing transformation from the tracking adapter local coordinate system into the biopsy tool's tip with the predefined long axis.
The main goal of this work was to assess the usefulness of OST HMD in the biopsy procedure according to the CT-based virtual plan. Additionally, we have calculated the accuracy of performed biopsies comparing results between trials using HMD and ED. For this purpose, each user performed 4 independent attempts accomplishing the same MP $(2 \times$ HMD + $2 \times$ ED). To verify the accuracy, we have applied the following procedure keeping in mind that each virtual biopsy was planned in such a way that the endpoint was located in the center of one of four holes. After each trial, the top part of the phantom's brain was removed, then, a digital image was acquired and metric calibrated to describe each pixel in physical units. The displacement between planned and performed biopsy's endpoint was measured in the following way:

1. each hole was detected on the photo as a circle using Hough transform,

2. the center of a circle from step 1 was determined to indicate VP planned biopsy location,

3. the performed biopsy endpoint was searched inside the circle from step 1 and its position was found.

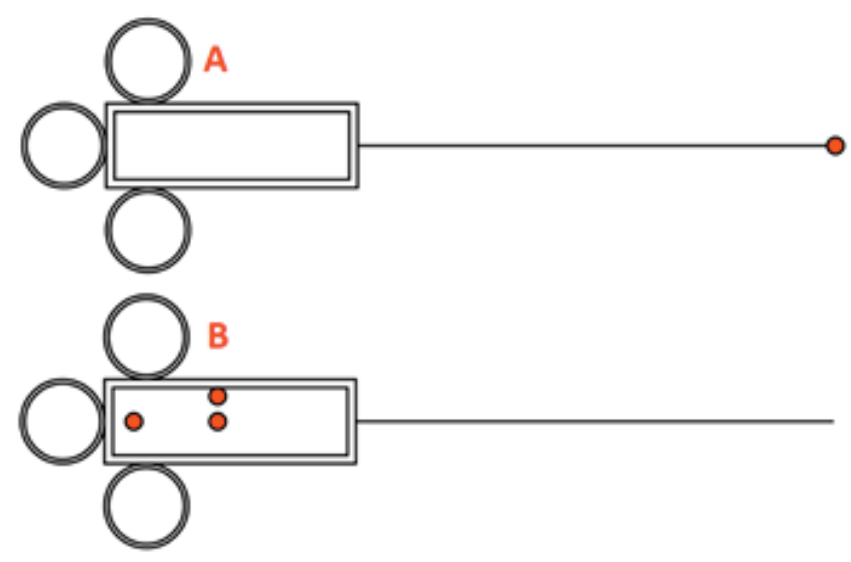

Fig. 7. Two-step biopsy tool calibration:

A) tool tip offset calculation, B) long axis determination 
One should keep in mind that the registration accuracy has a huge impact on the final biopsy results and OST HMD visualization accuracy. In the simplest case the registration outcome can be measured by the fiducial registration error (FRE) and the target registration error (TRE), and some predefined threshold can be used to inform if the surgery can be started.

\section{Results}

In Table 1, the averaged FRE and TRE errors for each user and trial are presented. Performed registration was considered successful when the mean FRE error was less than $1.50 \mathrm{~mm}$ while an averaged TRE error was less than $3.00 \mathrm{~mm}$, respectively. Since calculated registration errors were acceptable so biopsy procedure was performed in the next step. For each user and for all 48 trials $(2 \times 12$ using ED and $2 \times 12$ for HMD) biopsy result was satisfactory.

In Table 2, the calculated errors in [mm] between planned and performed biopsy are presented taking only the end biopsy point into account. For the first user, an average error from 24 trials using ED was 2.04 $\pm 0.83 \mathrm{~mm}$, while using HMD was $2.50 \pm 0.93 \mathrm{~mm}$. For the second user, reported errors were as follows: 2.69 $\pm 1.11 \mathrm{~mm}$ when applying ED and $2.17 \pm 0.82 \mathrm{~mm}$ with HMD support. We have also performed the Wilcoxon Signed-Rank test and Friedman's ANOVA test (with the threshold of statistical significance set at $\alpha<$ $0.05)$ to compare results obtained by users using ED versus HMD. For overall algorithm's comparison no statistical difference has been reported (with $p$-value $=$ 0.10 from Friedman's test) irrespective to biopsy mode or user's trial. Additionally, a greater repeatability was observed for ED mode than for HMD one. This can be explained by the fact that participants were more experienced in biopsy procedure using external displays while HMD mode was still in the learning phase.

\section{Discussion}

Biopsy error can be decomposed into three main components: VP image data to patient registration, OST HMD personal calibration, and finally, VP realization under the control of the navigation system. Previously, the biopsy procedure applied in MentorEye system was preliminary tested, including the abovementioned components except for visualization on OST HMD or personalized calibration. Initial verification was performed on phantom model equipped with tita-

Table 1. Averaged FRE and TRE errors in [mm] for each user and from each trial

\begin{tabular}{|c|c|c|c|c|c|c|c|c|}
\hline \multirow{2}{*}{} & \multicolumn{4}{|c|}{ User 1} & \multicolumn{4}{c|}{ User 2} \\
\cline { 2 - 9 } & \multicolumn{2}{|c|}{ ED } & \multicolumn{2}{c|}{ HMD } & \multicolumn{2}{c|}{ ED } & \multicolumn{2}{c|}{ HMD } \\
\cline { 2 - 9 } & $\# 1$ & $\# 2$ & $\# 1$ & $\# 2$ & $\# 1$ & $\# 2$ & $\# 1$ & $\# 2$ \\
\hline FRE $[\mathrm{mm}]$ & $1.10 \pm 0.50$ & $0.82 \pm 0.36$ & $0.95 \pm 0.32$ & $1.09 \pm 0.42$ & $1.01 \pm 0.34$ & $0.93 \pm 0.51$ & $1.02 \pm 0.29$ & $1.15 \pm 0.22$ \\
\hline TRE $[\mathrm{mm}]$ & $2.02 \pm 0.73$ & $2.14 \pm 0.68$ & $2.81 \pm 0.61$ & $2.17 \pm 0.86$ & $2.52 \pm 0.86$ & $2.12 \pm 0.41$ & $2.21 \pm 1.27$ & $2.87 \pm 0.68$ \\
\hline
\end{tabular}

Table 2. Calculated errors [mm] between planned and performed biopsy

\begin{tabular}{|c|c|c|c|c|c|c|c|c|}
\hline & \multicolumn{4}{|c|}{ User 1} & \multicolumn{4}{|c|}{ User 2} \\
\hline & \multicolumn{2}{|c|}{ ED } & \multicolumn{2}{|c|}{ HMD } & \multicolumn{2}{|c|}{ ED } & \multicolumn{2}{|c|}{ HMD } \\
\hline & $\# 1$ & $\# 2$ & $\# 1$ & $\# 2$ & $\# 1$ & $\# 2$ & $\# 1$ & $\# 2$ \\
\hline 1 & 1.49 & 1.56 & 0.95 & 3.60 & 0.32 & 1.20 & 0.16 & 3.22 \\
\hline 2 & 2.78 & 2.49 & 3.36 & 2.11 & 1.56 & 1.92 & 1.54 & 1.62 \\
\hline 3 & 1.08 & 3.62 & 3.63 & 1.67 & 1.99 & 4.04 & 1.88 & 2.00 \\
\hline 4 & 3.98 & 2.97 & 2.62 & 2.63 & 3.55 & 4.27 & 2.13 & 2.97 \\
\hline 5 & 1.55 & 1.38 & 0.64 & 0.97 & 2.13 & 4.05 & 1.20 & 2.19 \\
\hline 6 & 0.76 & 1.22 & 2.40 & 1.68 & 3.38 & 1.89 & 2.59 & 2.49 \\
\hline 7 & 2.28 & 1.43 & 3.65 & 3.08 & 2.34 & 1.30 & 2.80 & 2.77 \\
\hline 8 & 3.37 & 2.26 & 2.38 & 2.03 & 3.37 & 3.40 & 1.27 & 1.27 \\
\hline 9 & 1.59 & 1.85 & 1.77 & 1.68 & 1.78 & 2.40 & 1.50 & 2.97 \\
\hline 10 & 1.05 & 1.61 & 3.70 & 3.34 & 2.86 & 1.88 & 2.55 & 1.94 \\
\hline 11 & 1.88 & 2.42 & 3.05 & 2.94 & 2.50 & 3.81 & 3.34 & 2.84 \\
\hline 12 & 2.45 & 1.81 & 3.88 & 2.31 & 4.41 & 4.23 & 1.24 & 3.60 \\
\hline$p$-value & \multicolumn{2}{|c|}{0.695} & \multicolumn{2}{|c|}{0.084} & \multicolumn{2}{|c|}{0.347} & \multicolumn{2}{|c|}{0.110} \\
\hline \multirow{2}{*}{$\mathrm{Avg} \pm \mathrm{SD}$} & $2.02 \pm 0.94$ & $2.05 \pm 0.69$ & $2.67 \pm 1.04$ & $2.34 \pm 0.76$ & $2.52 \pm 1.04$ & $2.87 \pm 1.16$ & $1.85 \pm 0.84$ & $2.49 \pm 0.66$ \\
\hline & \multicolumn{2}{|c|}{$2.04 \pm 0.83$} & \multicolumn{2}{|c|}{$2.50 \pm 0.93$} & \multicolumn{2}{|c|}{$2.69 \pm 1.11$} & \multicolumn{2}{|c|}{$2.17 \pm 0.82$} \\
\hline
\end{tabular}


nium markers to enable better matching procedure. Obtained averaged error for entry point was $1.11 \pm$ $0.55 \mathrm{~mm}$ and $1.62 \pm 0.62 \mathrm{~mm}$ for stop point, while long axis deviation was determined as $0.37 \pm 0.12^{\circ}$. Detailed course of experiment was described in previous paper [16]. In the next step, stereoscopic visualization of VP using OST HMD was added to MentorEye system. Two personalized calibration procedures were implemented: one step SPAAM method, where both eyes are calibrated at the same time (described in the previous paper [30]) and two step SPAAM method, where both eyes are calibrated separately (applied in this study). Preliminary research on the accuracy of visualization method, presented in paper [30], has shown that obtained visualization accuracy is userdependent and correlated with previous experience with 3D technology. Furthermore, some problems with 3D perception were observed during one step SPAAM calibration procedure, which uses stereoscopic visualization. Therefore, two step SPAAM calibration procedure was recommended and implemented in MentorEye system. During our initial tests, we have confirmed that the accuracy of personalized calibration has a great impact on the navigated biopsy output. As a consequence, some specialized training must be introduced to increase user's domain knowledge about AR technology. Our initial trials have also revealed that AR content displayed on HMD should be minimized during surgery and strictly limited to the specific surgery navigation guides and color-coded information. Otherwise, the user is too focused on 3D content, and the misalignment between real and virtually generated objects significantly impairs visual perception inside the operating field of view.

Proper training and meticulously performed personalized calibration can ensure 3D virtual object perception at the acceptable accuracy level. This statement holds until HMD device is attached firmly and not moved in reference to user's head. Unfortunately, the surgery operating room is very dynamic, so HMD replacement can occur. In such a case, some recalibration procedures should be applied, but due to limited time, this is almost impossible. Taking all limitations described above into account, we have decided to introduce a biopsy guide and color-coded information instead of a whole 3D virtual object presentation. Two experienced users have tested the MentorEye biopsy navigation module using two different VP presentation methods: ED and OST-HMD, respectively. Personalized HMD calibration adds complexity to the whole surgical procedure. However, this burden is paid off in the intraoperative phase. Usage of HMD allows the user to focus on the surgery without losing a direct line of sight with the operating field of view as it happens for external displays. Both participants have reported that the proposed AR biopsy is userfriendly and easy to follow using OST HMD.

Our AR navigation system is based on infrared tracking (IR) technology. This fact is worth emphasizing as most of papers on similar surgery supporting systems seem to utilize two-dimensional (2D) opensource markers, with the quadratic markers being the most popular one [9]. IR tracking-based solutions, although relatively expensive, have been successfully integrated with medical devices and are used in clinical settings for decades. Great examples are neurosurgery and craniomaxillofacial surgery [5], [8]. These systems offer high accuracy (even up to $0.1 \mathrm{~mm}$ ) and working distance [14]. Moreover, their components are fully compatible with intraoperative requirements such as sterility or lighting conditions.

On the other hand, the experimental surgical systems, whose navigation is based on tracking of the 2D open-source fiducial markers, create a logistic challenge to meet hygiene requirements, such as the need to be printed on metal or covered with foil which may disturb detection. Another problem is the slow runtime of modern algorithms detecting marker systems at high resolutions (such as $2448 \times 2048$ ), rate lower than $30 \mathrm{fps}$ recommended for surgical AR-applications [8], the increase of the speed forcing the lowering of the resolution. Finally, the 2D fiducials seem to offer lower accuracy and reliability of the patient tracking process, which is the most decisive factor in its clinical applicability.

Notably, the hereby presented concept of biopsy support could also be adapted to aid precise bony structures drilling for the purpose of implant or fixation screws insertion, being an alternative for the standard intraoperative navigation systems or developed robotic devices [11].

\section{Conclusions}

In this paper, we have proposed and validated a two-step intra-operative module for navigated biopsy using an OST HMD device. In the first one, the user can perceive the whole VP content superimposed onto the surgery field of view. This is an introductory phase designed for initial biopsy adjustment. In the second stage, 3D models from VP are switched off and biopsy plan realization is based on a specially designed 3D surgery guide equipped with color-codes and textual data. It should be noted that the described 
algorithm provides good functionality and ergonomics being less dependent on the personalized HMD calibration accuracy and user's ability for an appropriate 3D depth perception. Despite enormous AR development, there are still some challenges to address. These are mainly connected with noticeable input latency and a small field of view, especially when HMD works in 3D mode. HMD can be successfully applied in CAS for less demanding tasks such as marker location or, as proposed, for biopsy supporting or for training purposes in which surgeon can check different VP scenarios. Future work will include more extensive tests using other HMD devices with higher display resolution to prove MentorEye clinical effectiveness.

\section{Acknowledgements}

The work is supported by National Centre of Research and Development in Poland, in frames of the project: "Development of Polish complementary system of molecular surgical navigation for tumor treatment ", STRATEGMED1/233624/4/NCBR/2014.

\section{References}

[1] Andrews C.M., Henry A.B., Soriano I.M., SOUTHWorth M.K., Silva J.R., Registration Techniques for Clinical Applications of Three-Dimensional Augmented Reality Devices, IEEE Journal of Translational Engineering in Health and Medicine, 2020, 9, DOI: https://doi.org/10.1109/JTEHM.2020.3045642.

[2] Badiali G., Cutolo F., Cercenelli L., M. Carbone M., D'AMATO R., FERRARI V., MARCHETTI C., The VOSTARS project: A new wearable hybrid video and optical see-through augmented reality surgical system for maxillofacial surgery, Int. J. Maxillofacial Surg., 2019, 48, DOI: https://doi.org/10.1016/ j.ijom.2019.03.472.

[3] Badiali G. et al., Review on Augmented Reality in Oral and Cranio-Maxillofacial Surgery: Toward "Surgery-Specific" Head-Up Displays, IEEE Access, 2020, 8, 59015-59028, DOI: 10.1109/ACCESS.2020.2973298.

[4] Bosc R., Fitoussi A., Hersant B., Dao T.H., Meningaud J.P, Intraoperative augmented reality with heads-up displays in maxillofacial surgery: A systematic review of the literature and a classification of relevant technologies, Int. J. Oral Maxillofacial Surg., 2019, 48 (1), 132-139, DOI: 10.1016/ j.ijom.2018.09.010.

[5] Carse B., Meadows B., Bowers R., Rowe P., Affordable clinical gait analysis: An assessment of the marker tracking accuracy of a new low-cost optical 3d motion analysis system, Physiotherapy, 2013, 99 (4), 347-351.

[6] Chen X., Xu L., Wang Y., Wang H., Wang F., Zeng X., WANG Q., EGGER J., Development of a surgical navigation system based on augmented reality using an optical see-through headmounted display, Journal of Biomedical Informatics, 2015, 55, DOI: https://doi.org/10.1016/j.jbi.2015.04.003.

[7] Cutolo F. et al., Ambiguity-Free Optical-Inertial Tracking for Augmented Reality Headsets, Sensors, 2020, 20 (5), DOI: $10.3390 / \mathrm{s} 20051444$.
[8] De Amici S., Sanna A., Lamberti F., Pralio B., A wii remote-based infrared-optical tracking system, Entertainment Computing, 2010, 1 (3-4), 119.

[9] Garrido-Jurado S., Munoz-Salinas R., Madrid-Cuevas F., MARIN-Jimenez M., Automatic generation and detection of highly reliable fiducial markers under occlusion, Pattern Recognition, 2014, 47 (6), 2280-2292.

[10] García-VÁzQuez V., Von HaXthausen F., JäcKLE S., SChumann C., Kuhlemann I., Bouchagiar J., Höfer A.C., Matysiak F., Hüttmann G., Goltz J.P., Kleemann M., ERNST F., HoRn M., Navigation and visualization with HoloLens in endovascular aortic repair, Innov. Surg. Sci., 2018, 3 (3), 167-177, DOI: 10.1515/iss-2018-2001.

[11] GIL J.J., DÍAZ I., ACCINI F., Inferring material properties in robotic bone drilling processes, Acta Bioeng. Biomech., 2019, 21 (3), 109-118, DOI: 10.5277/ABB-01386-2019-02.

[12] Grubert J., Itoh Y., Moser K., Swan J.E., A Survey of Calibration Methods for Optical See-Through Head-Mounted Displays, IEEE Transactions on Visualization and Computer Graphics, 2018, 24 (9), 2649-2662, DOI: 10.1109/ TVCG.2017.2754257.

[13] ITOH Y., KLINKER G., Interaction-free calibration for optical seethrough head-mounted displays based on 3D eye localization, Proc. IEEE Symp. 3D User Interfaces, 2014, 75-82.

[14] Kunz C., Genten V., Meirner P., Hein B., Metric-based evaluation of fiducial markers for medical procedures, Proc. SPIE 10951, Medical Imaging 2019: Image-Guided Procedures, Robotic Interventions, and Modeling, 109512O, 2019, DOI: https://doi.org/10.1117/12.2511720.

[15] Lin M.A., Siu A.F., BAE J.H., CUTKOSKy M.R., DANiEl B.L., HoloNeedle: Augmented reality guidance system for needle placement investigating the advantages of three-dimensional needle shape reconstruction, IEEE Robot. Autom. Lett., 2018, 3 (4), 4156-4162, DOI: 10.1109/LRA.2018.2863381

[16] Majak M., Żuk M., Świątek-NaJWer E., PoPeK M., PIETRUSKI P., Biopsy procedure applied in MentorEye molecular surgical navigation system, Lecture Notes in Computational Vision and Biomechanics, 2018, 27, 338-344.

[17] MAKIBUChI N., KATO H., YoneYAMA A., Vision-based robust calibration for optical see-through head-mounted displays, Proc. IEEE Int. Conf. Image Process., 2013, 2177-2181.

[18] MCKNight R.R., PEAN C.A., BuCK J.S. et al., Virtual Reality and Augmented Reality - Translating Surgical Training into Surgical Technique, Curr. Rev. Musculoskelet. Med., 2020, 13, 663-674, https://doi.org/10.1007/s12178-020-09667-3

[19] De Oliveira M.E., Debarba H.G., Lädermann A., Chagué S., CHARBONNIER C., A hand-eye calibration method for augmented reality applied to computer-assisted orthopedic surgery, Int. J. Med. Robot., 2019, 15 (2), DOI: 10.1002/rcs.1969.

[20] Pietruski P., Majak M., Świątek-Najwer E., Żuk M., PopeK M., MAZureK M., ŚWIECKA M., JAWOROWSKI J., Navigation-guided fibula free flap for mandibular reconstruction: A proof of concept study, Journal of Plastic, Reconstructive and Aesthetic Surgery, 2019, 72 (4), DOI: 10.1016/j.bjps.2019.01.026.

[21] Pietruski P., Majak M., Świątek-Najwer E., Żuk M., PopeK M., JAWOrowsKi J., MAZUREK M., Supporting fibula free flap harvest with augmented reality: A proof-of-concept study, The Laryngoscope, 2019, 130 (5), 1173-1179, https:// doi.org/10.1002/lary.28090

[22] Pietruski P. et. al., Supporting mandibular resection with intraoperative navigation utilizing augmented reality technology - A proof of concept study, Journal of Cranio-Maxillofacial 
Surgery, 2019, 47 (6), DOI: https://doi.org/10.1016/ j.jcms.2019.03.004.

[23] QIAN L., Barthel A., JohnSON A., Osgood G., KaZANZides P., NAVAB N., Fuerst B., Comparison of optical see-through head-mounted displays for surgical interventions with objectanchored 2D-display, Int. J. Comput. Assist. Radiol. Surg., 2017, 12 (6), DOI: 10.1007/s11548-017-1564-y.

[24] Rahman R., Wood M.E., Qian L., Price C.L., Johnson A.A., OsGood G.M., Head-Mounted Display Use in Surgery: A Systematic Review, Surgical Innovation, 2020, 27 (1), 88-100, DOI: $10.1177 / 1553350619871787$.

[25] Sakai D., Joyce K., Sugimoto M. et al., Augmented, virtual and mixed reality in spinal surgery: A real-world experience, Journal of Orthopaedic Surgery, 2020, DOI: 10.1177/ 2309499020952698.

[26] Tuceryan M., Genc Y., Navab N., Single-Point Active Alignment Method (SPAAM) for Optical See-Through HMD Calibration for Augmented Reality, Teleoperators and Virtual Environments, 2002, 11, 259-276.
[27] Wacker F.K., Vogt S.K., Khamene A., Jesberger J.A., Nour S.G., Elgort D.R., SAUER F., Duerk J.L., LEWIN J.S., An augmented reality system for MR image-guided needle biopsy: initial results in a swine model, Radiology, 2006, 238 (2), 497-504.

[28] Wang H., Wang F., Xu L., Chen X., Wang Q., Precision insertion of percutaneous sacroiliac screws using a novel augmented reality-based navigation system: a pilot study, International Orthopaedics, 2016, 40, 1941-1947.

[29] Wang J., Shen Y., YANG S., A practical marker-less image registration method for augmented reality oral and maxillofacial surgery, Int. J. Comput. Assist. Radiol. Surg., 2019, 14 (5), 763-773, DOI: 10.1007/s11548-019-01921-5.

[30] ŻUK M., Majak M., ŚwiąTEK-Najwer E., PopeK M., KULAS Z., Evaluation of calibration procedure for stereoscopic visualization using optical See-Through Head Mounted Displays for a complex oncological treatment, Lecture Notes in Computational Vision and Biomechanics, 2018, 27, 354-359. 